\title{
Silicon Strip Detectors for the ATLAS HL-LHC Upgrade
}

\section{Jose Bernabeu*i}

IFIC - Instituto de Fisica Corpuscular (University of Valencia and CSIC),

Edificio Institutos de Investigacion, Apartado de Correos 22085, E-46071 Valencia, Spain

E-mail: Pepe.Bernabeulific.uv.es

\begin{abstract}
While the Large Hadron Collider (LHC) at CERN is continuing to deliver an ever-increasing luminosity to the experiments, plans for an upgraded machine called High Luminosity LHC (HLLHC) are progressing. The upgrade is foreseen to increase the LHC design luminosity by a factor ten. The ATLAS experiment will need to build a new tracker for HL-LHC operation, which needs to be suited to the harsh HL-LHC conditions in terms of particle rates and radiation doses. In order to cope with the increase in pile-up backgrounds at the higher luminosity, an all silicon detector is being designed. New radiation-hard prototype silicon sensors have been produced for the strip region of the future ATLAS tracker in a $\mathrm{n}^{+}$-strip p-type option. The sensors have been irradiated beyond the expected fluences in the HL-LHC collider and tested with prototype readout electronics. This paper summarizes the design and radiation performance of the $\mathrm{n}^{+}$-strip $\mathrm{p}$-type sensors.
\end{abstract}

10th International Conference on Large Scale Applications and Radiation Hardness of Semiconductor Detectors

6-8 July 2011

Florence, Italy

\footnotetext{
*Speaker.

${ }^{\dagger}$ On behalf of the ATLAS Upgrade Community.
} 


\section{Introduction}

The Large Hadron Collider (LHC) is providing proton-proton collisions with a continuous increase in the luminosity delivered to the different experiments. Physics data is being recorded by the ATLAS [1] experiment, which is designed to fully exploit the phyics potential of the LHC up to its nominal luminosity of $10^{34} \mathrm{~cm}^{-2} \mathrm{~s}^{-1}$. It is planned to extend the LHC physics program by increasing the instantaneous luminosity by one order of magnitude around 2022 (Fig.1) in an upgraded machine called High Luminosity LHC (HL-LHC). With a target of $\sim 3000 \mathrm{fb}^{-1}$ total recorded integrated luminosity per experiment, the HL-LHC will allow to improve the precision in different measurements within the Standard Model, enlarge the discovery region of particles predicted by some supersymmetric theories and enhance the sensitivity to low-rate phenomena inaccessible at the LHC scale [2].

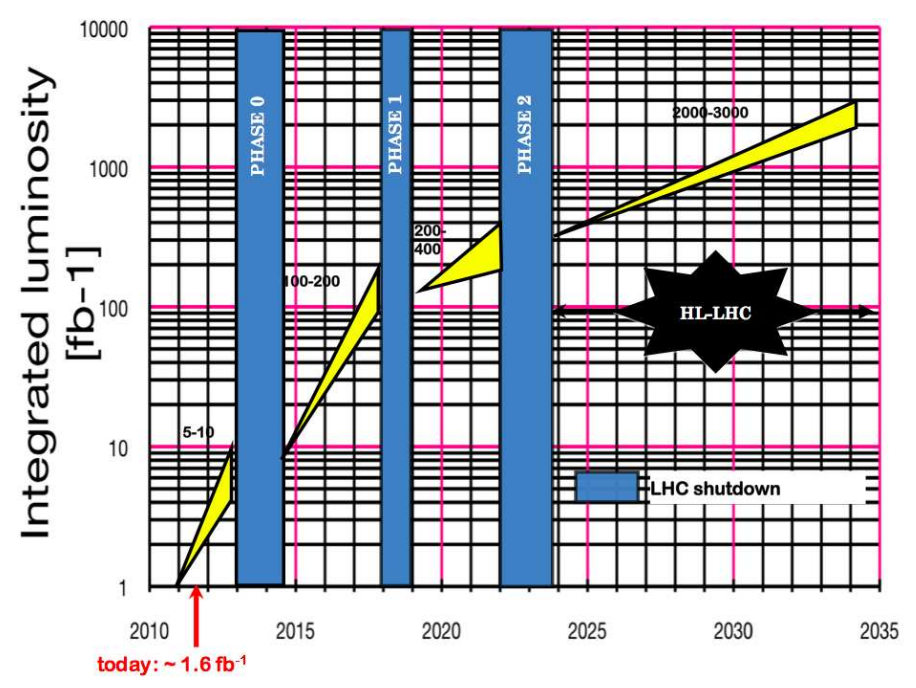

Figure 1: LHC plans.

\subsection{New ATLAS Inner Tracker}

The expected luminosity at the HL-LHC will increase the number of pile-up interactions per crossing from $\sim 23$ (as predicted in the current LHC at the design luminosity) up to $\sim 200$. The current tracker will not be able to stand the cumulated radiation damage and it will not provide the required tracking performance due to the huge increase in the channel occupancy. Therefore, a completely new inner tracker must be designed and built for the HL-LHC operation. The two main requirements are: on one side much better granularity in order to be able to distinguish the tracks of the different particles, on the other side it needs to have much better radiation hardness.

Most probably, the future ATLAS tracker will be an all silicon-based system. Figure 2 shows the current "straw-man" layout. The central barrel region consists of four pixel layers and five strip layers. The three innermost layer have short strips $(2.48 \mathrm{~cm} \mathrm{long}$ ) and the outer two layer have long strips ( $4.8 \mathrm{~cm}$ long). The forward region is covered by six pixel and five strip disks. This layout ensures 9-hit coverage in the pseudo-rapidity range $|\eta| \leq 2.5$. 


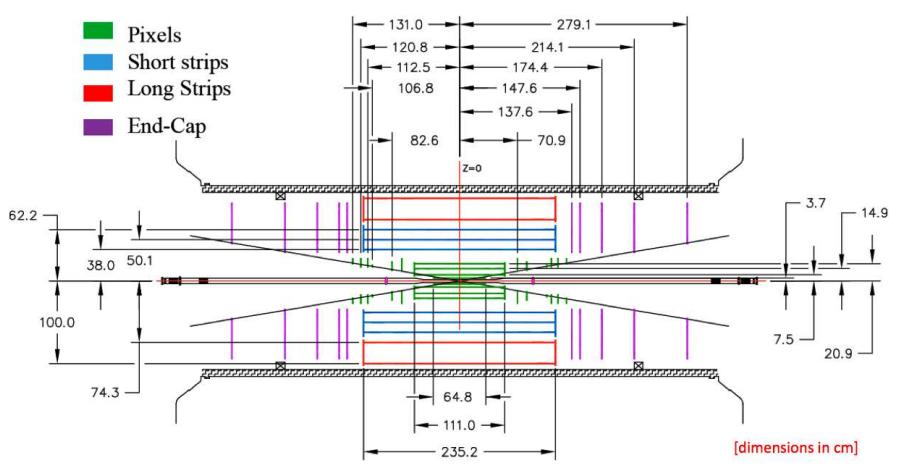

Figure 2: Straw-man layout (v14) for the future ATLAS tracker.

The expected fluence as function of the radial distance to the beamline for the "straw-man" layout and a total integrated luminosity of $3000 \mathrm{fb}^{-1}$ is shown in Fig.3. The maximum expected fluence in the strip barrel area is $1.2 \times 10^{15} \mathrm{n}_{e q} / \mathrm{cm}^{2}$, corresponding to the innermost short strip layer at $\mathrm{Z}=0$. This number includes a safety factor of two. The first barrel long-strip layer expects $5.6 \times 10^{14} \mathrm{n}_{e q} / \mathrm{cm}^{2}$. For the strip forward region, the maximum expected fluence of $1.2 \times 10^{15}$ $\mathrm{n}_{e q} / \mathrm{cm}^{2}$ corresponds to the intermediate third disk at the lowest radii.

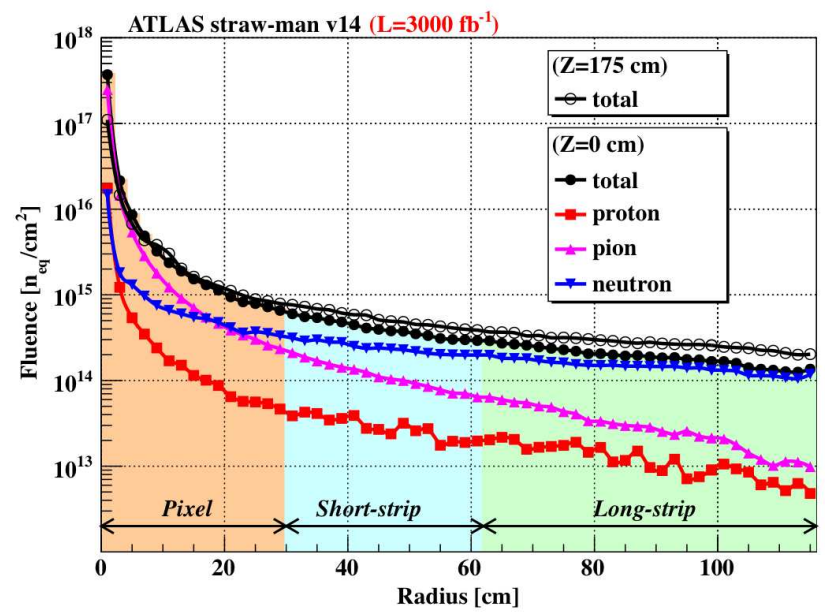

Figure 3: Expected fluences as function of the radial distance to the beamline for $3000 \mathrm{fb}^{-1}$. Two cases are shown, $\mathrm{Z}=0 \mathrm{~cm}$ and $\mathrm{Z}=175 \mathrm{~cm}$.

\section{Strip Tracker Design}

Several R\&D projects are well advanced in terms of design and assembly of strip modules integrating sensors, hybrids with readout electronics, cooling and services. Figure 4 shows two possible integration concepts for the short-strip barrel region. In the baseline stave concept, a common mechanical structure integrates the sensors, the electrical lines (bus cable) and cooling circuit. The stave has a central core composed of a spacing material (carbon-foam or honeycomb) and carbon fiber facings glued on both sides. Silicon microstrip detectors are glued on the bus 
cable and hybrids carrying the front-end electronics are then glued on top of the sensitive side of the detectors. In the fallback super-module concept, individual double-sided silicon strip modules are assembled into a light-weighted local support structure. Each module is composed by a central base-board, two silicon sensors and four hybrids with readout electronics.

Prototypes, in both options, of modules with prototype electronics, sensors and assembly components have been constructed and electronically tested. Assemblies of modules in short staves or supermodules $(40 \mathrm{~cm}$ instead of the final $120 \mathrm{~cm}$ ) have been produced and tested.

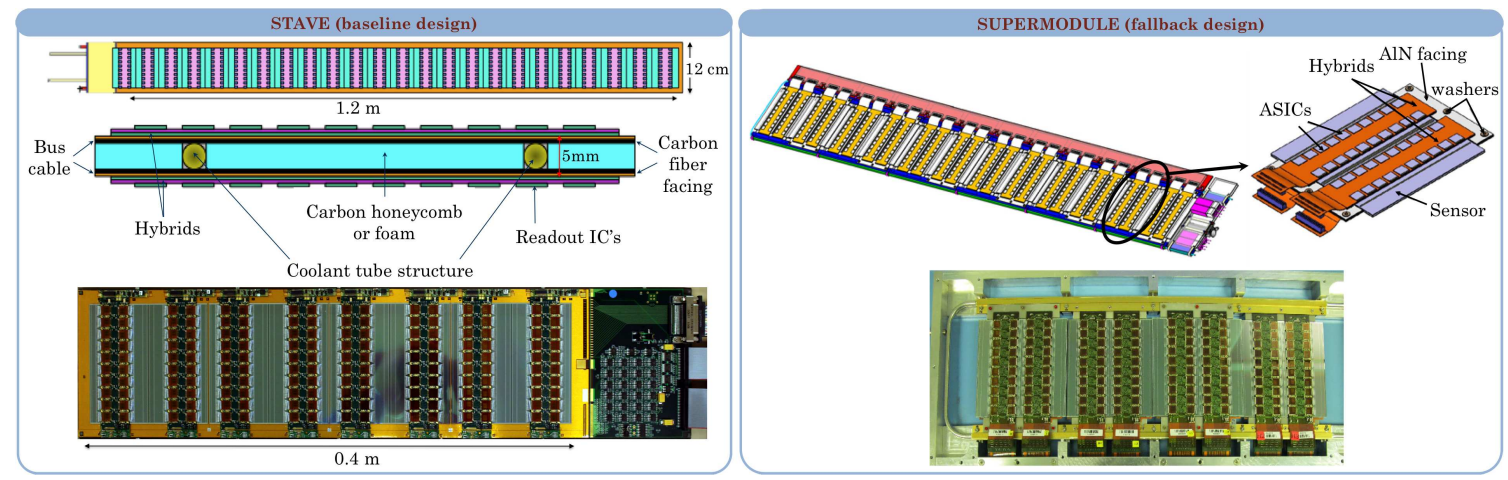

Figure 4: Stave (left) and super-module (right) barrel strip integration concepts.

\section{Silicon Sensors}

Silicon sensors for the new tracker have been designed, the choice of $\mathrm{n}^{+}$strips in $\mathrm{p}$ type substrate was found to be more adequate. With n-strips, electrons are collected, thus, the signal is faster and reduces the effect of trapping centres produced by the high level of radiation. P-type substrate permits to have a single sided process during manufacturing, a major advance compared to having a $\mathrm{n}$-type substrate (in the $\mathrm{n}^{+}$-in-n option) which requires an expensive manufacture processing on both sides. In addition, the selected $\mathrm{n}^{+}$-in-p option depletes always from the strip side, permitting to work underdepleted.

A large area $\mathrm{n}^{+}$-in-p silicon strip sensor has been developed by the ATLAS collaboration and produced by Hamamatsu Photonics in a 6-inch $(150 \mathrm{~mm})$ wafer [3]. The mask layout of the sensor is shown in Fig. 5. The detector area is $97.54 \times 97.54 \mathrm{~mm}^{2}$ and its thickness is $320 \mu \mathrm{m}$. The bulk is p-type float zone silicon with a lattice orientation $\left\langle\begin{array}{llll}1 & 0 & 0\end{array}\right\rangle$. The $\mathrm{n}^{+}$readout strips are AC-coupled and have polysilicon bias resistors. The isolation structure is p-stop. The sensor has four $2.39 \mathrm{~cm}$ strip segments, two with axial strips parallel to the sensor edges and two with stereo strips tilted at an angle of $40 \mathrm{mrad}$. The reason of this tilt is that when sensors are assembled back to back, an axial strip on one side matches a stereo strip on the other side, giving not only $\phi$ information, but also Z. The strip pitch is $74.5 \mu \mathrm{m}$ and there are a total of 1280 strips per segment.

In addition to the main sensor, $2410 \times 10 \mathrm{~mm}^{2}$ miniature sensors are included in the wafer. The miniature sensors have 104 strips of eight mm length and implement different surface strip isolation structures (p-stop) and punch-through protection (PTP) structures. 


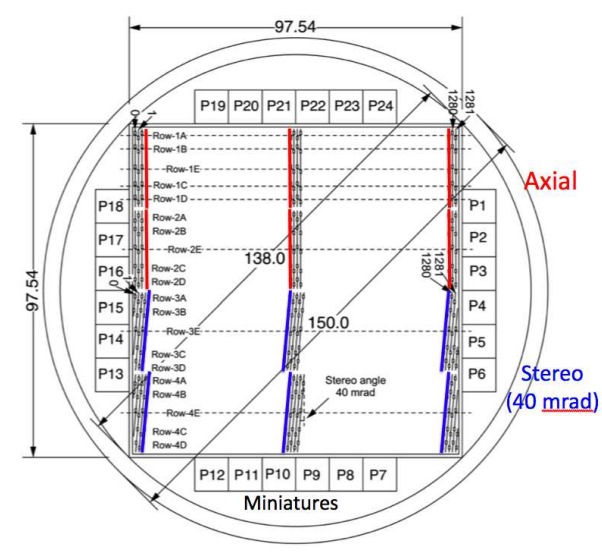

Figure 5: Mask layout of the barrel strip sensor prototypes.

\section{Measurements on Full Size Sensors}

The full size sensors have been extensively tested before irradiation. Both the bulk and strip characteristics have been thoroughly evaluated to check the sensors were effectively compliant with the required technical specifications before irradiation [4]. Figure 6 shows the comparison of the different specifications and the measured parameters, all within specifications. As an example, the depletion voltage measurement is shown.

\begin{tabular}{|l|c|c|}
\hline Parameter & Specification & Measurement \\
\hline Leakage Current & $<200 \mu \mathrm{A} @ 600 \mathrm{~V}$ & $200 \mathrm{nA}-370 \mathrm{nA}$ \\
\hline Depletion Voltage & $<500 \mathrm{~V}$ & $190 \mathrm{~V}-245 \mathrm{~V}$ \\
\hline Interstrip Capacitance & $<1.1 \mathrm{pF} / \mathrm{cm}$ (3probes) & $0.7 \mathrm{pF} / \mathrm{cm}$ \\
\hline Coupling Capacitance & $>20 \mathrm{pF} / \mathrm{cm}$ & $24-30 \mathrm{pF} / \mathrm{cm}$ \\
\hline Polysilicon Resistance & $1.5 \pm 0.5 \mathrm{M} \Omega$ & $1.3-1.6 \mathrm{M} \Omega$ \\
\hline Interstrip Resistance & $>10 \mathrm{xRbias} \approx 15 \mathrm{M} \Omega$ & $>19 \mathrm{G} \Omega$ \\
\hline
\end{tabular}

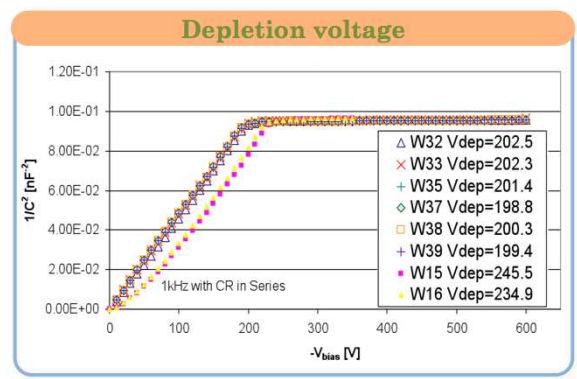

Figure 6: Values of the pre-radiation full size sensor specifications and measurements (left). Depletion voltage measurement for different sensors (right).

Modules for the two design concepts have been built with these full size sensors. Assembled with prototype electronics, calibration tests show good module performance with $\sim 600$ electron noise for both design concepts. One module was irradiated in the PS facility at CERN, the $24 \mathrm{GeV}$ proton beam scanned the full module area. The module was biased, powered and clocked during the irradiation up to $1.9 \times 10^{15} \mathrm{n}_{e q} / \mathrm{cm}^{2}$. After irradiation the module was fully functional and the noise slightly increased by $10 \%$, well in agreement with predicted electronic simulations.

\section{Measurements on Miniature Sensors}

Miniature sensor have been mainly used for irradiation studies. They have been irradiated with protons, pions and neutrons at different irradiation facilities. The doses were all up to at least $1 \times 10^{15} \mathrm{n}_{e q} / \mathrm{cm}^{2}$, in a few irraditions the sensors were irradiated up to $1 \times 10^{16} \mathrm{n}_{e q} / \mathrm{cm}^{2}$. The 
IV-characteristics, the depletion voltage, the interstrip capacitance and resistance and the charge collection efficiency have been measured [5].

The irradiated sensors were distributed among different testing laboratories to check the charge collection. Very good agreement was found in the measurements among the different samples (Fig. 7), which is a good indication for the measurement validity as each laboratory uses a complete different data acquisition system. A signal to noise $(\mathrm{S} / \mathrm{N})$ ratio of $\sim 15$ was found after $1 \times 10^{15}$ $\mathrm{n}_{e q} / \mathrm{cm}^{2}$ irradiation with $500 \mathrm{~V}$ of sensor bias, well above the required specification of 10 . Collected charge as function of the fluence show a good safety margin (Fig. 7). Also, it is worth to notice the good agreement of results among the different particle irradiations.
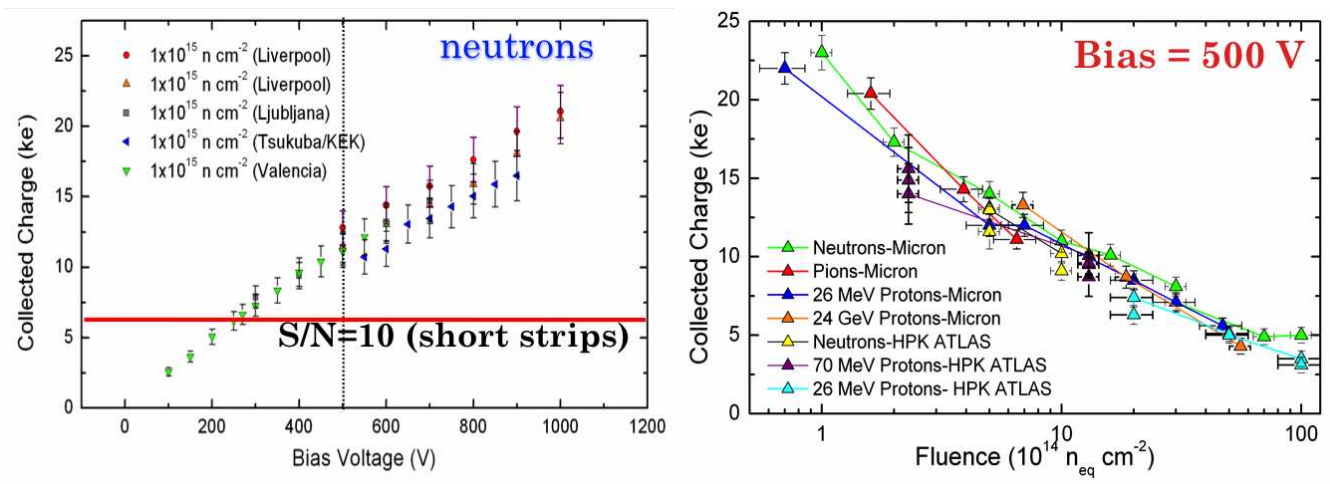

Figure 7: Collected charge of neutron irradiated miniature sensors as a function of the bias voltage (left). $\mathrm{S} / \mathrm{N}$ is above specification. Collected charge of irradiated miniature sensors in different facilities as function of the fluence (right).

For heavily irradiated sensors $\left(>5 \times 10^{15} \mathrm{n}_{e q} / \mathrm{cm}^{2}\right.$,), it has been observed that the signal charge increases with higher bias voltage [6] (Fig. 8). This phenomenon has already been seen in other semiconductor sensors and it is known as "charge multiplication". It happens at very high local electric fields, likely due to the impact ionisation by the electrons being collected. The multiplication gain (M) is low, well away from "Geiger mode" operation. Thus, multiplication is still in proportional regime. M certainly depends on voltage, the collected charge versus bias voltage is over-linear. An interesting field is opened here as the sensors could be used at higher fluences, so they are more radiation hard. Also, the possibility of using thin sensors appears, as for the same bias voltage the electric fields are higher.

Miniature sensors have been as well used to test the so-called Punch Through Protection (PTP) structure. In case of a beam loss by a beam accident, the beam could directly point to the silicon sensor. In this case, an enourmous amount of charge would be created in the silicon bulk. The amount of free carriers would temporarily collapse the electric field distribution, with the result of a large voltage appearing at the implant strip (Fig. 8). As the aluminium readout strip is grounded through the electronics, a high voltage difference is developed between the implant strip and the readout strip. The coupling capacitance could break, and even more the spike current going to the electronics could destroy it.

In order to protect the sensor and the electronics, miniature sensors include PTP structures of different designs (Fig. 8). When high voltage appears at the implant strip, the PTP structure would conduct the current towards the bias ring, protecting the coupling capacitor. First results [7] show 


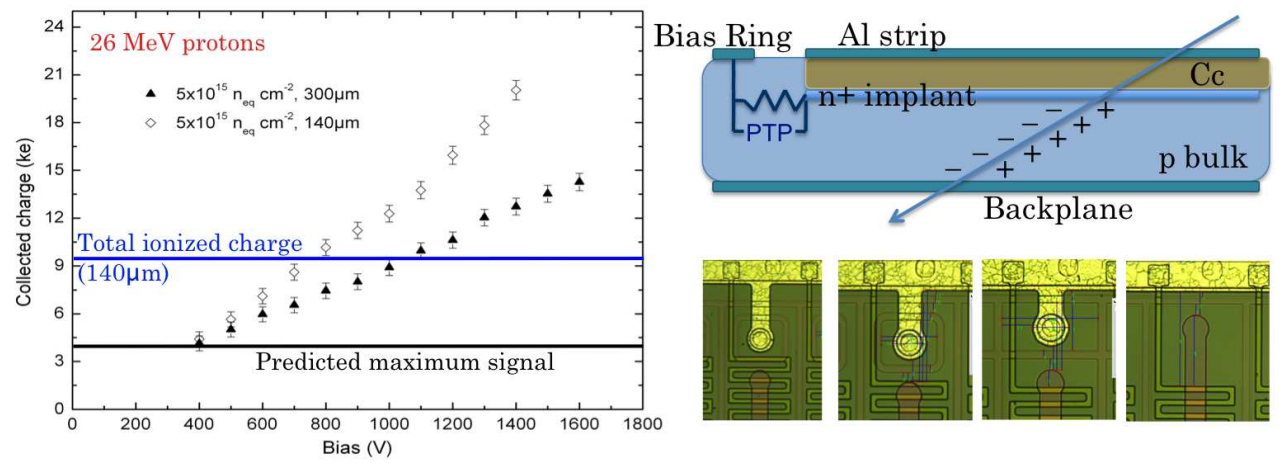

Figure 8: Collected charge vs bias voltage for heavily irradiated miniature sensors (left). There is charge multiplication as collected charge can be higher than the ionized. Diagram of a silicon sensor showing the PTP location (top right). Different PTP structures in miniature sensors, where the implant strip is close to the bias ring (bottom right).

that the structures behave as expected and that they are radiation hard. More studies are ongoing to understand this complex mechanism.

\section{Endcap Sensors}

The development program for the endcap strip integration is based on adapting the barrel stave option to a petal shape. The sensor will use the same technology as the barrel ones. The geometry of the endcap is complicated as it needs six rings with six different sensors (Fig. 9). In addition, the pitch has a great variation (67 to $106 \mu \mathrm{m}$ ) which leads to high bonding angles if no pitch adapter is used. The area at lower radii is quite component-congested and pitch adapters hardly fit. Another geometrical problem is that in order to use the same sensors on both sides of the petal, the strips are not pointing to the center of the disk, so the strips are not parallel to the sensor edge and some strips are truncated.
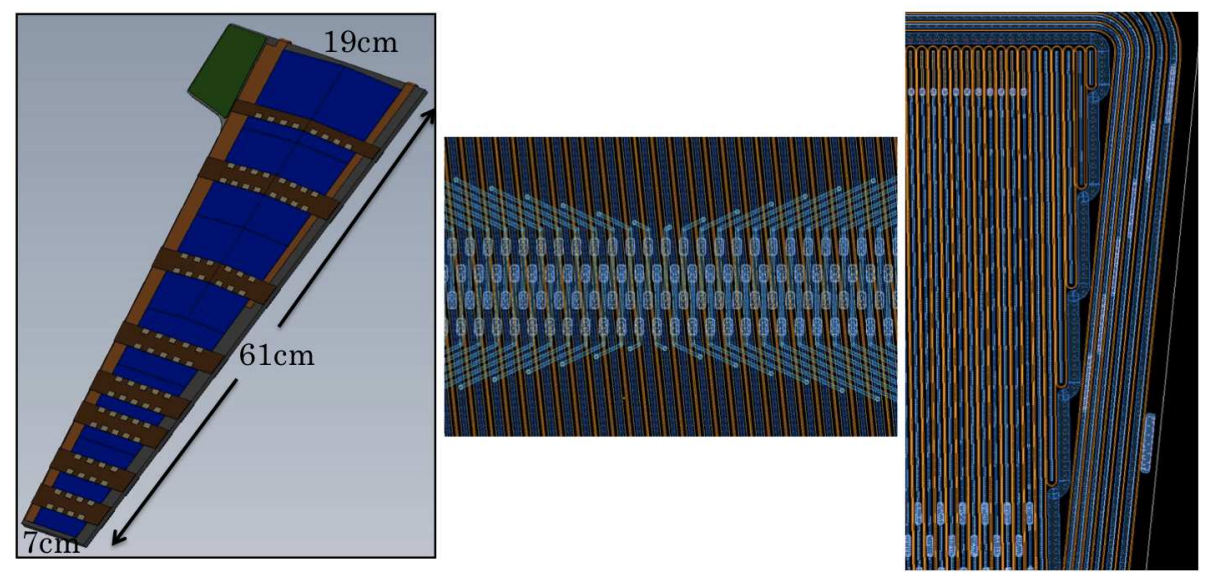

Figure 9: Petal layout (left). Embedded pitch adapter (center). Truncated edge strips (right). 
Some designs have been proposed and, in order to test them, smaller sensors (from 4-inch wafers) have been designed (Fig. 9) and are currently under production. Small petals (petalets) will test these solutions.

\section{Conclusions}

The present ATLAS tracker will be replaced by a new all-silicon tracker. Sensors for this tracker have been produced in $\mathrm{n}^{+}$-in-p technology, their performance is already very good as they fullfill final specifications. Modules and stavelets have been constructed with these sensors, achieving very good performance. Sensors and modules are fully functional after irradiation at the expected dose in HL-LHC. Miniature sensors have been produced, which permitted to verify radiation hardness and test punch through protection structures against beam accidents. Charge multiplication has been observed in miniature sensors after irradiation. The endcap strip sensors are being designed, and smaller size prototype fabrication in ongoing.

\section{References}

[1] The ATLAS Collaboration (G. Aad et al.), The ATLAS Experiment at the CERN Large Hadron Collider, Journ. of Instrum. 3 (2008).

[2] K. Jakobs, Physics at the LHC and sLHC, Nucl. Instr. and Meth. A 636 (2011) S1-S7.

[3] Y. Unno et al., Development of n-in-p silicon sensors for very high radiation environments, Nucl. Instr. and Meth. A 636 (2011) S24-S30.

[4] J. Bohm et al., Evaluation of the bulk and strip characteristics of large area $n$-in-p silicon sensors intenteded for a very high radiation environment, Nucl. Instr. and Meth. A 636 (2011) S104-S110.

[5] K. Hara et al., Testing of bulk radiation damage of n-in-p silicon sensors for very high radiation environments, Nucl. Instr. and Meth. A 636 (2011) S83-S89.

[6] G. Casse et al., Evidence of enhanced signal response at high bias voltages in planar silicon detectors irradiated up to $2.2 \times 10^{16} \mathrm{n}_{e q} / \mathrm{cm}^{2}$, Nucl. Instr. and Meth. A 636 (2011) S56-S61.

[7] S. Lindgren et al., Testing of surface properties pre-rad and post-rad of n-in-p silicon sensors for very high radiation environments, Nucl. Instr. and Meth. A 636 (2011) S111-S117. 